\title{
Stability and synthesis of superheavy elements: Fighting the battle against fission - example of ${ }^{254}$ No
}

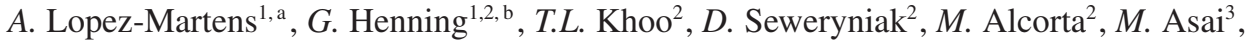

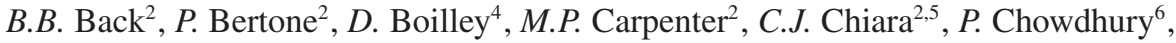

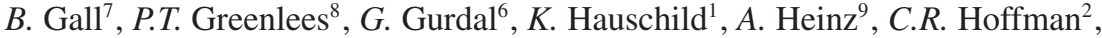

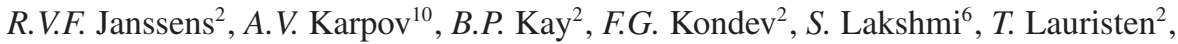 \\ C.J. Lister ${ }^{6}$, E.A. McCutchan ${ }^{2}, C$. Nair ${ }^{2}, J$. Piot $^{7, \mathrm{c}}, D$. Potterveld ${ }^{2}, P$. Reiter ${ }^{11}$, \\ $N$. Rowley ${ }^{12}, A . M$. Rogers ${ }^{2}$, and $S . \mathrm{Zhu}^{2}$ \\ ${ }^{1}$ CSNSM, IN2P3/CNRS and Université Paris Sud, France \\ ${ }^{2}$ Argonne National Laboratory, USA \\ ${ }^{3}$ Japan Atomic Energy Agency, Japan \\ ${ }^{4}$ GANIL, CEA and IN2P3/CNRS and Normandie Université, France \\ ${ }^{5}$ University of Maryland, USA \\ ${ }^{6}$ Univeristy of Massachusetts Lowell, USA \\ ${ }^{7}$ IPHC, IN2P3/CNRS and Université Louis Pasteur, France \\ ${ }^{8}$ University of Jyväskylä, Finland \\ ${ }^{9}$ Chalmers Tekniska Högskola, Sweden \\ ${ }^{10}$ Flerov Laboratory of Nuclear Reactions, JINR, Russia \\ ${ }^{11}$ Universität zu Köln, Germany \\ ${ }^{12}$ IPN Orsay, IN2P3/CNRS and Université Paris Sud, France
}

\begin{abstract}
Superheavy nuclei exist solely due to quantum shell effects, which create a pocket in the potential-energy surface of the nucleus, thus providing a barrier against spontaneous fission. Determining the height of the fission barrier and its angular-momentum dependence is important to quantify the role that microscopic shell corrections play in enhancing and extending the limits of nuclear stability. In this talk, the first measurement of a fission barrier in the very heavy nucleus ${ }^{254}$ No will be presented.
\end{abstract}

\section{Introduction}

The nucleus of interest, ${ }^{254} \mathrm{No}$, is situated at the very top of the nuclear chart in the region of transfermium nuclei. These nuclei are characterized by a decreasing, and for the heaviest nuclei, a vanishing liquid-drop fission barrier. If ${ }^{254}$ No was just as a liquid drop of incompressible fluid consisting of 102 positively-charged protons and 152 neutrons, its fission barrier would be of the order of $0.9 \mathrm{MeV}$ [1]. This means that ${ }^{254} \mathrm{No}$ would fission with a lifetime of less than a nanosecond. Experimentally, however, we know that the half life of ${ }^{254} \mathrm{No}$ is $51 \mathrm{~s}$ and that its main mode of decay is $\alpha$ decay. Since its production cross section

\footnotetext{
a e-mail: araceli.lopez-martens@csnsm.in2p3.fr

${ }^{\mathrm{b}}$ Current address: IPHC, Strasbourg

${ }^{\mathrm{c}}$ Current address: GANIL, Caen
} 


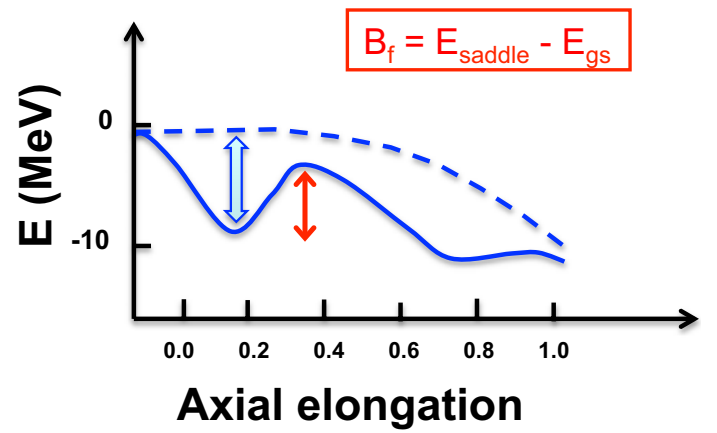

Figure 1. Potential energy of the nucleus as a function of axial deformation. The dashed line shows the liquid drop prediction for ${ }^{264} \mathrm{Hs}(\mathrm{Z}=108)$. The solid line shows the effect of shell corrections on the potential energy.

is one of the highest in the mass region, it is also a nucleus that has been extensively studied via prompt spectroscopy [2-5]. These studies have revealed that ${ }^{254}$ No is deformed and emits a spectrum of photons and conversion electrons, typical of regular rotational motion. ${ }^{254}$ No is in fact very robust against fission and has been observed up to spin $24 \hbar$.

The reason why ${ }^{254}$ No has an enhanced stability against fission is due to the fermionic nature of the neutrons and protons in the nucleus, which occupy discrete energy levels grouped into shells. It is the regions of low level density, or gaps in the single-particle energy spectra, which give additional binding to the nucleus: the ground-state is lowered with respect to the liquid drop energy and a barrier against fission appears. As illustrated in Fig. 1, the height of the barrier $B_{f}$ is defined as the energy difference between the ground state and the highest point on the path to fission: the saddle point. This barrier is what enables the existence of the heaviest nuclei. This makes it a valuable quantity to study - though technically it is not an observable one can measure directly.

Different theoretical models predict different barrier heights. So measuring $B_{f}$ also provides a test of theories. In a macroscopic-microscopic approach where the liquid drop part of the potential is calculated using the Finite Range Liquid Drop Model (FRLDM) and the folded Yukawa potential is used for the microscopic shell correction, the barrier for ${ }^{254} \mathrm{No}$ is predicted to be $6.7 \mathrm{MeV}$ [6]. In a fully microscopic approach, J.L. Egido and L.M. Robledo used the Gogny effective interaction and the Hartree Fock Bogoliubov approximation to compute the potential energy surface of the nucleus as a function of axial deformation for different values of total angular momentum [7]. A barrier of $8.66 \mathrm{MeV}$ is obtained. What this model can also tell us is how the barrier evolves with spin. In accordance with experimental observations, the self-consistent calculations predict that the barrier will survive to quite high spin.

How the barrier behaves as a function of spin, in other words the moment of inertia of the saddle point configuration, is important to study because if the barrier were to disappear at a finite spin, the heaviest elements could not be synthesized. Indeed, one has to keep in mind that these heavy nuclei are produced in fusion-evaporation reactions, which bring in a substantial amount of angular momentum. The cross section to produce a given evaporation residue depends on three terms: (i) The probability that the colliding projectile and target will penetrate inside the entrance channel potential barrier and reach the contact point, (ii) the probability that this capture state evolves into a fused compound nucleus, and finally, (iii) the probability that the excited compound nucleus evaporates neutrons and decays to the ground state of the nucleus of interest while surviving fission every step of the way. This survival 

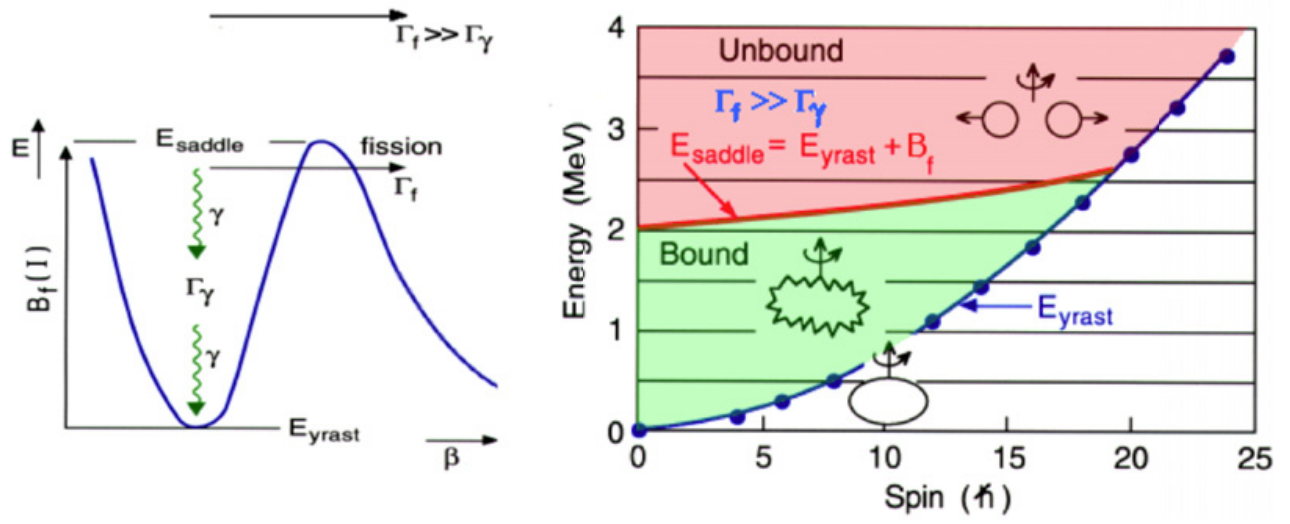

Figure 2. Left: schematic plot of the potential energy of the nucleus as a function of axial deformation illustrating how $\gamma$ decay dominates below the saddle-point energy while fission takes over at energies above the barrier. Right: cartoon showing how the competition between $\gamma$ emission and fission splits the excitation energy vs. spin plane into two regions.

probability reflects the competition between neutron emission, fission and $\gamma$ decay and one of the key parameters is the fission barrier, which enters into the fission width $\Gamma_{f}$. In fact, the high fission barriers predicted around $Z=114$ and $N=184$ are thought to be responsible for reversing (at $Z=112$ ) the trend of ever decreasing evaporation-residue cross sections as a function of atomic number $Z$, resulting in a maximum effect at $Z=114-115$ [8]. One should also note that factors, such as dissipation, may also contribute to reduce $\Gamma_{f}$ [9].

Experimentally, no fission barriers are known above $\mathrm{Bk}(Z=99)$. The probability to fission has been measured in direct reactions on actinide targets [10,11], by electromagnetic excitation of secondary beams following fragmentation reactions [12] and in a few cases the electron capture (EC) delayed fission branch was used to determine the height of the fission barrier [13]. Unfortunately, these methods are not easily applicable to the transfermium region. The two first methods cannot be used because of lack of suitable targets and primary beams. And the third method is limited to the cases where EC populates states around and above the fission barrier in the daughter nucleus.

\section{Entry distribution method}

A different approach for determining the fission barrier was proposed by P. Reiter more than ten years ago [14]. It is called the entry distribution method and has the advantage that it is the only way to study the spin dependence of the barrier. As is shown in Fig. 2, it relies on the fact that in the cases where the fission barrier lies below the neutron separation energy, fission is the dominant process above the barrier. This means that the excitation energy vs. spin plane can be divided into two regions above the yrast line: a region where the nucleus is bound and $\gamma$ decays to the ground state and a region above the saddle point, where the nucleus fissions. In order to probe the saddle-point line separating the two regions, the idea is to measure the distribution of total energy and total spin of the ${ }^{254}$ No nucleus right after neutron evaporation. As more energy is put into the system in the fusion reaction, one should be able to observe the distribution move up in energy and spin and be eventually cut off by fission. The distribution falls to half its maximum value at the energy at which the fission and $\gamma$-decay modes compete with equal probability. This energy is called the 1/2 energy and differs from the saddle point energy by a small factor $\Delta(I)$. A recent analysis has shown that the model dependency of $\Delta$ is small [15]. 
The entry distribution of ${ }^{254} \mathrm{No}$ was measured by Reiter et al. using the reaction ${ }^{208} \mathrm{~Pb}\left({ }^{48} \mathrm{Ca}, 2 n\right)$ at two different bombarding energies: 215 and $219 \mathrm{MeV}$. At the lowest energy, it was found that the distribution was limited by the maximum energy available in the reaction. At the higher bombarding energy, no cut off of the entry distribution was observed, and so only a lower limit of $5 \mathrm{MeV}$ for the fission barrier at $12 \hbar$ could be extracted [14]. The experiment, however, showed that the bulk of the cross section to produce ${ }^{254}$ No occurs at high spin.

\section{Experiment}

The experiment was repeated in order to probe the entry distribution at higher beam energy: 219 and $223 \mathrm{MeV}$. The $10 \mathrm{pnA}{ }^{48} \mathrm{Ca}$ was delivered by the Argonne Tandem Linac Accelerator System. ${ }^{254}$ No was produced at the target position of the Fragment Mass Analyzer (FMA) [16] which separated the evaporation residues from the other unwanted reaction products. The recoiling ${ }^{254}$ No ions were identified at the focal plane of the FMA while $\gamma$ rays emitted by the excited ${ }^{254}$ No were detected by GAMMASPHERE [17], which was used in its calorimetric mode. In such a mode, the anti-Compton BGO shields surrounding the Ge detectors are also used to detect photons allowing for an efficiency of $\sim 78 \%$ to be reached [18]. The response function of GAMMASPHERE was measured with radioactive sources so that the distribution of emitted photon energies and multiplicities could be reconstituted from the measured total energies and number of firing detector modules. This is achieved via an iterative unfolding procedure $[19,20]$ starting with an initial guess at the emitted distribution. This trial distribution is then passed through the measured response function and compared to the measured distribution. In doing so, an improved guess at the true distribution can be made and the whole procedure can be repeated. To get to the total energy vs. spin distribution, one has to know a little bit more about the nucleus of interest, in particular the contributions of conversion electrons to the total spin and energy. These parameters can be obtained and/or extracted from the known level scheme or from experimental spectra. In the case of ${ }^{254} \mathrm{No}$, this was made possible by extensive prompt and decay spectroscopy performed by many groups worldwide [2, 3, 5, 21-24]. The average spin and energy removed by conversion electrons obtained from the known level decay scheme of ${ }^{254}$ No and tabulated in reference [15] is in excellent agreement with what was measured by Butler et al. [4] before the level scheme was known, giving confidence in our understanding of the average decay cascade in ${ }^{254} \mathrm{No}$. The entry distributions obtained at 219 and $223 \mathrm{MeV}$ bombarding energies are shown in Fig. 3. The crosses indicate the points where the distributions fall to half of their maximum value. What is immediately noticeable is that even though more spin and energy $(4 \mathrm{MeV})$ are put into the system between the left and right panels of Fig. 3, the crosses between 12 and $20 \hbar$ remain at constant excitation energy, $E^{*}$. This saturation is attributed to the effect of fission depleting the $\gamma$-emitting states. The saddle point energies extracted from the saturated half-maximum points yield a moment of inertia of the saddle of 125(60) $\mathrm{MeV}^{-1}$. This is in good agreement with what can be extracted from the self-consistent potential energy surfaces of Ref. [7]. The value of the barrier height at spin $15 \hbar$ is found to be $B_{f}=6.0(5) \mathrm{MeV}$. Extrapolating down to zero spin using the saddle point moment of inertia, one finds $B_{f}(0)=6.6(9) \mathrm{MeV}$. This value of the excitation energy above which fission dominates the decay process is in good agreement with the predictions of Möller et al. [6]. An interesting thing to note is that a $6.6 \mathrm{MeV}$ barrier is larger than what one would naively obtain by adding the liquid drop barrier height and the amplitude of the microscopic shell correction in ${ }^{254}$ No [25]: $B_{f_{0}}=0.9+3.97=4.9 \mathrm{MeV}$. This implies a positive shell effect of more than $1.5 \mathrm{MeV}$ at the saddle point. Another point to mention is that the measured average spin distribution of 


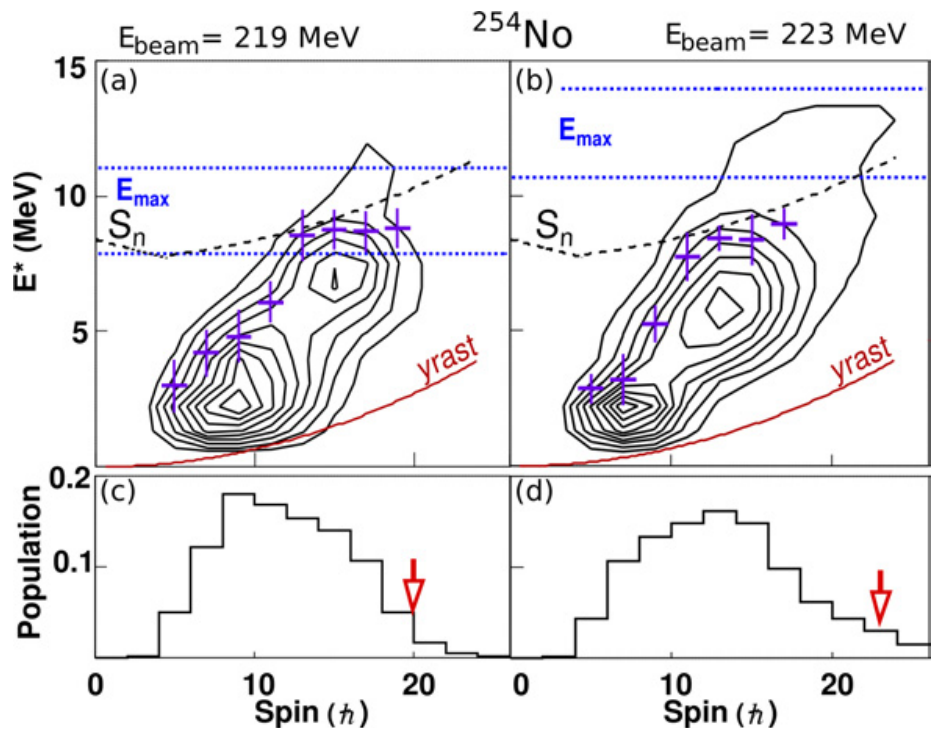

Figure 3. Plot of the entry distribution of ${ }^{254}$ No measured at 219 (left) and 223 (right) MeV bombarding energies and their corresponding projections onto the spin axis. The yrast line and neutron separation energy are shown together with the interval of maximal energy available in the reaction. The crosses represent the half-maximum energies at a given spin value. The points at high spin have been discarded as they originate from events resulting from random summing. The red arrows point to the spins at which the distribution falls by one tenth of its maximum value.

the surviving ${ }^{254}$ No is shifted towards higher spins by nearly $\sim 5 \hbar$ compared to calculations (e.g., see Fig. 6 of Ref. [26]).

\section{Conclusions and perspectives}

This is the first time that the excitation energy at which fission starts to dominate the decay process has been determined in a transfermium nucleus. The results obtained and extrapolated to spin zero are in agreement with macroscopic-microscopic calculations of the barrier height. This may not be so surprising since these calculations are the only ones which reproduce the single-particle energy spectra in this mass region [27].

Extending the method to other nuclei requires the knowledge of their average decay schemes. This should be made possible soon by the coupling of a new generation $\gamma$-ray spectrometers, such as AGATA (Advanced GAmma Tracking Array) [28], GRETINA (Gamma Ray Energy Tracking In beam Nuclear Array) [29] and also digital GAMMASPHERE to powerful spectrometers such as VAMOS (VAriable MOde Spectrometer) gas-filled [30] and AGFA (Argonne Gas Fille Analyzer) [31]. Decay schemes of nuclei around ${ }^{254}$ No will also be established and/or improved by decay spectroscopy studies performed with increased beam intensities and more efficient decay stations. These technical developments should allow the investigation of the average fission barrier height in odd systems and also the magnitude of the barrier associated to particular configurations of the nucleus (e.g. high- $K$ states) for which the hindrance towards fission is expected to be large.

Finally, measuring the entry distribution of evaporation residues is useful in itself as it can be used as a starting point to Monte Carlo simulations of the decay flow to the ground 
state and compared to experiment in order to get a handle on level densities and $\gamma$ strength functions in super heavy nuclei.

\section{References}

[1] A.J. Sierk, Phys. Rev. C 33, 2039 (1986).

[2] M. Leino et al., Eur. Phys. J. A 6, 63 (1999).

[3] P. Reiter et al., Phys. Rev. Lett. 82, 509 (1999).

[4] P.A. Butler et al. Phys. Rev. Lett. 89, 202501(2002).

[5] S. Eeckhaudt et al., Eur. Phys. J. A 26, 227 (2005).

[6] P. Möller et al., Phys. Rev. C 79, 064304 (2009).

[7] J.L. Egido and L.M. Robledo, Phys. Rev. Lett. 85, 1198 (2000).

[8] Y. Oganessian, Nucl. Phys. News Vol. 23, 15 (2013).

[9] R. Yanez et al., Phys. Rev. Lett. 112, 152702 (2014).

[10] B.B. Back et al., Phys. Rev. C 9, 1924 (1974).

[11] B.B. Back et al., Phys. Rev. C 10, 1948 (1974).

[12] A. Grewe et al., Nucl. Phys. A614, 400 (1997).

[13] D. Habs et al., Z. Phys. A285, 53 (1978).

[14] P. Reiter et al., Phys. Rev. Lett. 84, 3542 (2000).

[15] G. Henning et al., Phys. Rev. Lett. 113, 262505 (2014).

[16] C.N. Davids et al., Nucl. Instr. Meth. B70, 358 (1992).

[17] I.Y. Lee, Nucl. Phys. A 520, 641c (1990).

[18] T. Lauritsen et al., Phys. Rev. C 75, 064309 (2007).

[19] M. Jääskeläinen et al., Nucl. Instr. Meth. 204, 385 (1983).

[20] P. Benet, PhD thesis, Université Louis Pasteur, Strasbourg (1988).

[21] R.-D. Herzberg et al., Nature 442, 896 (2006).

[22] S. Tandel et al., Phys. Rev. Lett. 97, 082502 (2006).

[23] F.P. Heßberger et al., Eur. Phys. J. A 43, 55 (2010).

[24] R.M. Clark et al., Phys. Lett. B 690, 19 (2010).

[25] P. Möller, J. Nix, W. Myers, W. Swiatecki, At. Data Nucl. Data Tables 59, 185 (1995).

[26] V.I. Zagrebaev et al., Phys. Rev. C 65, 014607 (2001).

[27] D. Seweryniak et al. Nucl. Phys. A834, 357c (2010).

[28] S. Akkoyun, et al., Nucl. Instr. Meth. A668, 26 (2012).

[29] S. Paschalis, et al., Nucl. Instr. Meth. A709, 44 (2013).

[30] Ch. Schmitt et al., Nucl. Instr. Meth. A621, 558 (2010).

[31] B.B. Back et al., Proposal for the Argonne Gas Filled Analyzer - AGFA (2013). 